\title{
Evaluation of the Secretome Profile and Functional Characteristics of Human Bone Marrow Mesenchymal Stromal Cells-Derived Conditioned Medium Suggest Potential for Skin Rejuvenation
}

\author{
Sudha Balasubramanian ${ }^{*}$, Charan Thej ${ }^{1,2^{*}}$, Ankita Walvekar ${ }^{1}$, Priyanka Swamynathan ${ }^{1}$, \\ Pawan K. Gupta1, Raviraja N. Seetharam1 ${ }^{1}$, Anish S. Majumdar ${ }^{1 \#}$ \\ ${ }^{1}$ Stempeutics Research Pvt. Ltd., Bangalore, India \\ ${ }^{2}$ Manipal University, Manipal, India \\ Email: balasubramanian.sudha@gmail.com, charan.thej@stempeutics.com, ankita.walvekar@gmail.com, \\ priyanka.swamynathan@gmail.com,pawan.gupta@stempeutics.com,raviraja.ns@stempeutics.com, \\ \#anish.majumdar@stempeutics.com
}

How to cite this paper: Balasubramanian, S., Thej, C., Walvekar, A., Swamynathan, P., Gupta, P.K., Seetharam, R.N. and Majumdar, A.S. (2017) Evaluation of the Secretome Profile and Functional Characteristics of Human Bone Marrow Mesenchymal Stromal Cells-Derived Conditioned Medium Suggest Potential for Skin Rejuvenation. Journal of Cosmetics, Dermatological Sciences and Applications, 7, 99-117.

https://doi.org/10.4236/jcdsa.2017.71010

Received: February 13, 2017

Accepted: March 19, 2017

Published: March 22, 2017

Copyright (C) 2017 by authors and Scientific Research Publishing Inc. This work is licensed under the Creative Commons Attribution International License (CC BY 4.0)

http://creativecommons.org/licenses/by/4.0/

(c) (7) Open Access

\begin{abstract}
Background/Purpose: Multipotent bone marrow-derived mesenchymal stromal cells (BMMSC) have been shown to possess the potential for tissue regeneration. The application of mesenchymal stromal cells (MSC)-derived growth factors and cytokines (GF/CKs) has been implicated for the repair and regeneration of the damaged skin that occurs due to aging and exposure to environmental stress factors. Methods: We have used both qualitative and quantitative measurements of the GF/CKs from the conditioned medium (CM) of a pooled population of BMMSC by antibody array analysis as well as by enzyme-linked immunosorbent assay (ELISA). Furthermore, the CM was also used in a variety of in vitro biological assays to measure its protective properties in human skin fibroblasts. Results: We have characterized the secretome of BMMSC by analyzing the composition of the CM using a forty-one growth factor array system. Thirteen of these GF/CK/extra cellular matrix (ECM)/ matrix metalloproteinases (MMP)-inhibitors in the CM were quantified owing to their involvement in skin repair cascade. In addition, we report that the BMMSC-CM was also able to protect dermal fibroblasts against tert-Butyl hydro peroxide (tbOH) induced oxidative stress and ultra violet $\mathrm{B}$ (UV-B) radiation induced cell damage. Conclusion: Based on the data presented here, we propose that BMMSC-derived CM may have the potential to promote health and rejuvenation of the aging skin.
\end{abstract}




\section{Keywords}

Bone Marrow-Derived MSC, Conditioned Medium, Secretome, Oxidative Stress, UV-B Damage

\section{Introduction}

Skin is the outermost protective layer of the human body and hence any damage caused to it is quite visible. Skin damage and skin aging may be caused due to a combination of various intrinsic factors, such as chronological aging or extrinsic factors, such as ultraviolet (UV) rays (photo-aging) and other environmental stressors [1]. Aging of the skin at the cellular level caused due to these factors, is manifested by the development of sagging skin, onset of wrinkling, appearance of blemishes or age spots, altered pigmentation or loss of skin tone and hydration [1] [2] [3]. These changes may progressively increase, leading to serious skin health problems [2]. Various medical treatments and topical cosmeceuticals have been used to treat some symptoms of photo-aging; however, the results have been less than satisfactory [3]. The advances made in the past decades have been associated with clinical, biochemical and molecular changes of dermal aging which has led to the development of various products intended to reduce, delay, and in some cases, repair the untoward effects of programmed ageing and external environmental damage [4]. Topical application of pharmacological formulations with anti-aging properties, laser therapy, radiofrequency treatments, use of skin peels and invasive methods like introducing dermal fillers or cosmetic surgery are some of the current treatments employed to reverse the effect of aging [2] [5].

The emergence of stem cell therapy and greater understanding of the biology of these cells, have opened up avenues to develop novel stem cell based products for regenerative medicine. Among the many types of stem cells characterized until recently, mesenchymal stromal cells (MSC) has been the preferred cell type for use in therapy primarily due to their anti-inflammatory/immunomodulatory and regenerative properties. These cells have been obtained and characterized from many tissues like bone marrow, adipose, umbilical cord and umbilical cord blood, placenta, amnion, dental pulp and others [6]. One of the characteristics specific to MSCs is their potential to differentiate into adipocytes, chondrocytes and osteocytes as well as to cells belonging to other lineages. Elucidation of the mechanism of action of MSCs to heal and regenerate a given damaged tissue points largely to the trophic factors secreted by these cells, both in vitro and in vivo [7]. Furthermore, the secretome of MSC contains a large array of growth factors, cytokines, chemokines, enzymes, extracellular matrix proteins and small molecules [5]. These factors possess the ability to trigger a cascade of biological reactions such as cell proliferation and migration, tissue granulation and epithelialization and angiogenesis [8]. As a result, research in this area has been intensified so as to carefully examine the proteomic profile and functional potency of 
MSC derived CM in order to explore the possibility of its potential use to develop anti-aging therapy [9] [10].

Although several types of MSCs have been shown to possess skin-regenerative properties, it has been reported that cytokines obtained from BMMSC are preferred for formulating topical anti-aging products for the skin, compared to the factors obtained from MSCs of other tissue sources [2]. We had earlier reported and published the development and characterization of a pooled, bone marrow derived MSC population, obtained from healthy, consenting donors, that were expanded in serum-containing or serum-free medium in a reproducible manner in a GMP compliant facility [11] [12] [13].

Recently, CM based regenerative approach has been explored for cosmeceutical applications as it contains a wide range of factors, which are secreted by MSCs isolated from various tissues [14] [15]. These growth factors and cytokines present in the $\mathrm{CM}$, are believed to be beneficial in reducing signs of skin aging, owing to their capacity to promote dermal fibroblast and keratinocyte proliferation, and in inducing extracellular matrix component production, including deposition and reorganization of collagen, thereby promoting skin health [2] [16].

Encouraged by the recent results, we set out to characterize the CM from pooled, human BMMSC cultures during the cell expansion stage. The rationale for using a three donor pool of BMMSC as opposed to a single donor derived cells relates to the fact that MSCs including BMMSC from different donors show significant variability in their proteomic signature which may eventually be manifested in the composition of the CM [17] [18] [19]; and one of the ways to minimize this problem would be to use a pooled population of MSCs for CM production. In this communication, we report that this medium is highly enriched in factors that are known to stimulate cell proliferation and migration, angiogenesis, remodeling of ECM and re-epithelialization, and a combination of such diverse macro- and small molecules may inversely modulate the skin aging process. A series of functional assays, designed to determine the beneficial effect of the $\mathrm{CM}$ were performed using fibroblasts in vitro to determine if such $\mathrm{CM}$ derived from BMMSC can minimize, slow the progress and even reverse some of the aging related skin symptoms induced by oxidative stress and radiation exposure. Our results suggest that topical application of a skin formulation containing such growth factor-rich CM, obtained from pooled BMMSCs, as the active ingredient, may aid in skin rejuvenation and provide anti-aging effects.

\section{Materials and Methods}

\subsection{BMMSC Culture and CM Collection}

BMMSCs from three different healthy human volunteer donors were pooled in equal proportions and cultured in $1 \times$ Dulbecco's Modified Eagle's MediumKnockout $^{\mathrm{TM}}$ (DMEM-KO (GIBCO, Carlsbad, USA) containing inorganic salts, amino acids, vitamins and glucose, and supplemented with $10 \%$ fetal bovine serum (FBS) (Hyclone, Waltham, MA, USA), 1× Penstrep (GIBCO, Carlsbad, USA), $1 \times$ Glutamax $^{\text {TM }}$ (GIBCO, Carlsbad, USA) and $2 \mathrm{ng} / \mathrm{ml}$ bFGF (Sigma- 
Aldrich, St. Louis, Missouri, USA) and incubated in a $5 \% \mathrm{CO}_{2}$ incubator (Binder, Germany) at $37^{\circ} \mathrm{C}$ [11]. These cells were cultured up to Passage 5. At passage 5 , the CM from the cells was collected at the time of harvest and the cell debris was removed by centrifugation at $1500 \mathrm{rpm}$, for 5 minutes, at room temperature. One portion of the CM was concentrated 10 times $(10 \times)$ with Millipore $1 \mathrm{KDa}$ or $3 \mathrm{KDa}$ cut-off filters using the Tangential Flow Filtration (TFF) technology (Merck-Millipore, NJ, USA). The remaining unconcentrated CM and the 10x $\mathrm{CM}$ were stored at $-80^{\circ} \mathrm{C}$ until used for subsequent analysis. Similarly, the control medium (complete medium without BMMSC exposure) was also concentrated 10 times and stored at $-80^{\circ} \mathrm{C}$ until use. The $1 \times \mathrm{CM}$, concentrated $10 \times \mathrm{CM}$ and their respective controls were used at various dilutions that were made using serum-free medium (SFM), for all the assays. SFM was considered as negative control in all the experiments. A complete workflow of the process of collection of the CM, composition of proteomic analytes and quantification of a selected group of GF/CKs and functional assays pertaining to skin health is described in Figure 1.

\subsection{Semi Quantitative Analysis of BMMSC Secretome by Chemiluminescence Detection}

The CM was analyzed for the presence of cytokines, chemokines and growth factors by performing a semi-quantitative human growth factor antibody based array (RayBio ${ }^{\circledast}$, Norcross GA, USA). The experiment was performed as per

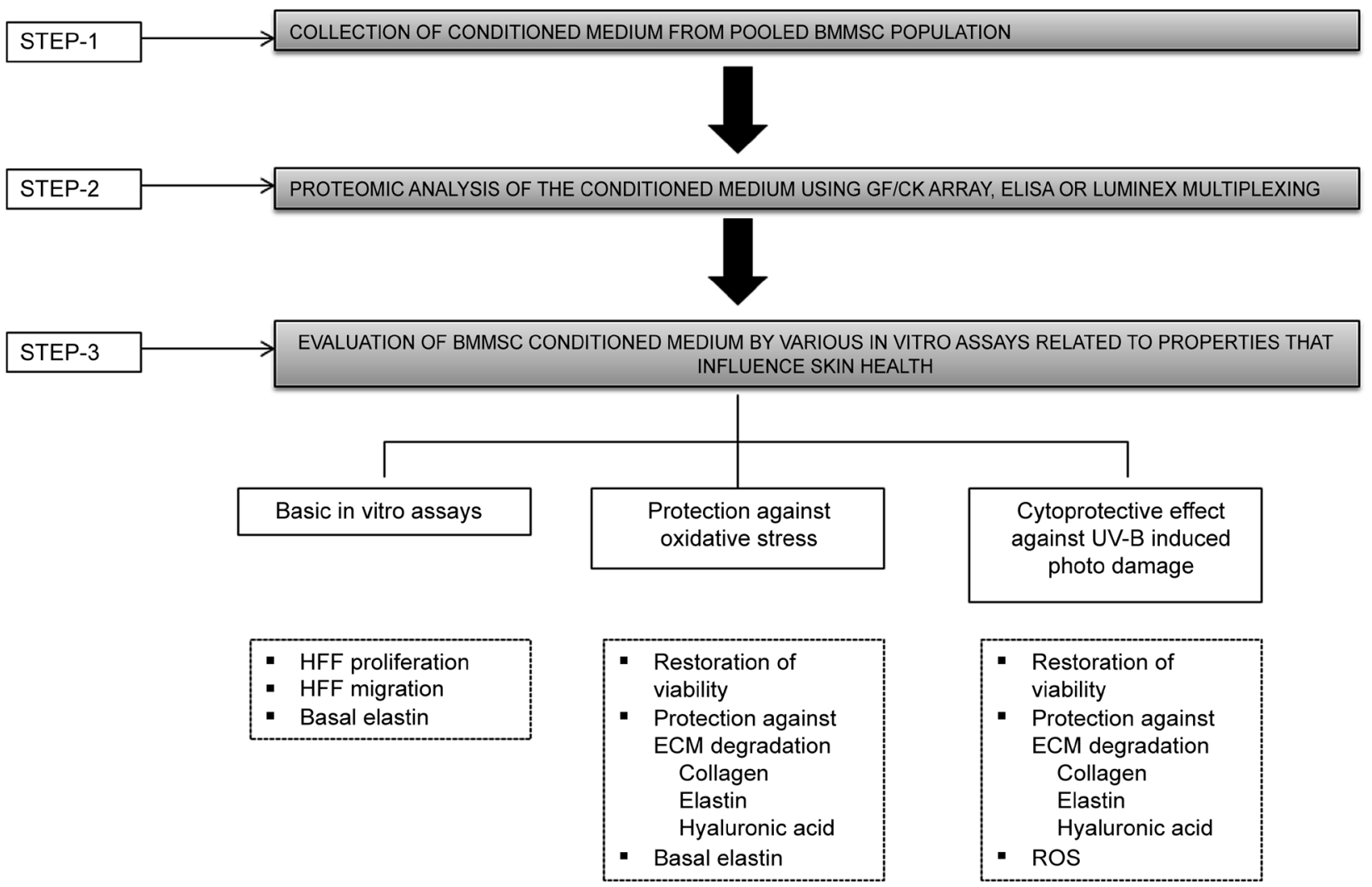

Figure 1. Flow chart indicating the experimental design of the study. 
manufacturers' instructions and chemiluminescence was recorded using Image Quant LAS 4000 (GE Healthcare, Little Chalfont, UK). The data recorded was analyzed using Image J software (NIH). The relative intensities of individual growth factors were calculated as arbitrary units after background correction and normalized to control media blot intensities.

\subsection{Quantification of Human Cytokines or Growth Factors in the CM}

To quantify a select group of protein macro-molecules in the CM, human specific ELISA kits (R \& D systems, Minneapolis, USA) were used, following the manufacturer's instructions, or multiplexing was carried out using Millipore's proprietary Luminex ${ }^{\oplus}$ Technologies which uses internally color coded microspheres with two fluorescent dyes mixed at various ratios such that each bead has its own unique identity. Each of these beads is tagged with specific capture antibodies. The analytes in the test samples and standards was captured by the tagged beads after which a secondary biotinylated detection antibody was introduced. This reaction mixture was then incubated with streptavidin PE conjugate which acted as the reporter. The microspheres are allowed to pass through two lasers one that excites the bead and the other that excites the fluorophore PE. The reading was captured on Luminex $200^{\mathrm{TM}}$ (Austin, TX, USA) and based on their relative position and intensities, values were generated. The values in picograms/milliliter $(\mathrm{pg} / \mathrm{mL})$ so obtained were interpolated from the standard graph generated by the in-built system software.

\subsection{Human Foreskin Fibroblast Cell Culture}

Human Foreskin Fibroblast-1 cell line, HFF-1 (Promocell, Heidelberg, Germany), were cultured in $1 \times$ DMEM supplemented with 10\% FBS (HI Media, Mumbai, India), $1 \times$ Penstrep and $1 \times$ GlutamaxTM and incubated in a $5 \% \mathrm{CO}_{2}$ incubator at $37^{\circ} \mathrm{C}$ and $95 \%$ humidity. The cells were serum starved with $1 \times$ DMEM supplemented with $0.1 \%$ FBS, $1 \times$ Penstrep, and $1 \mathrm{X} \times$ GlutamaxTM and incubated for 24 hours prior to being used in further assays.

\subsection{Migration Assay}

HFF- 1 cells were seeded at a density of $2500 \mathrm{cells} / \mathrm{cm}^{2}$ in a 6 well plate and cultured for 24 hours, in 10\% FBS containing DMEM medium, under $5 \% \mathrm{CO}_{2}$ conditions, at $37^{\circ} \mathrm{C}$ and $95 \%$ humidity, to achieve approximately $90 \%$ confluence. The cells were then serum starved for 24 hours in DMEM containing $0.1 \%$ FBS, after which, a scratch (wound) was created using a $200 \mu \mathrm{l}$ pipette tip. The cells were then treated with varying concentrations of $1 \times \mathrm{CM}$ or concentrated $10 \times$ $\mathrm{CM}$. Images were captured at $0 \mathrm{~h}$ and $24 \mathrm{~h}$ time points, and the distance of wound closure was measured using Image J software (NIH).

\subsection{Proliferation Assay}

HFF-1 cells were seeded at a density of 2000 cells per well in a 96 well micro-titer 
plate. On the following day, the cells were serum starved for $24 \mathrm{~h}$, after which they were treated with various concentrations of CM. Forty eight hours after the addition of CM or control medium, the cells were pulsed with BrdU (2000 fold dilution in SFM) from the BrdU kit (Merck-Millipore, NJ, USA). Twenty four hours post BrdU pulsing, the cells were further processed as per the manufacturer's instructions. Percentage proliferation of HFF-1 cells was calculated from the OD values obtained using the Spectramax M3 plate reader (Molecular Devices, CA, USA).

\subsection{Estimation of Elastin Levels}

Fifty thousand HFF cells per well were seeded in a 24 well plate. Cells were serum starved for $24 \mathrm{~h}$ after which the CM or the control medium was added. After $72 \mathrm{~h}$ of incubation, the basal levels of elastin were estimated in the culture supernatant by ELISA (Cusabio, MD, USA).

\subsection{Cyto-Protection}

Two thousand HFF cells per well were seeded in a 96 well plate. The cells were serum starved for $24 \mathrm{~h}$, after which they were treated with $300 \mu \mathrm{M}$ of tbOH for 2 $\mathrm{h}$ or photo-damaged by UVB irradiation $\left(230 \mathrm{~mJ} / \mathrm{cm}^{2}\right)$. The condition medium and the control medium at various dilutions were then added onto the cells and incubated for $48 \mathrm{~h}$, after which MTT assay was performed and the OD values were recorded at $405 \mathrm{~nm}$.

\subsection{Protection of Elastin and Collagen Degradation in Response to Oxidative/UV-B Damage}

Fifty thousand HFF cells per well were seeded in a 24 well plate. The HFF-1 cells were treated with $300 \mu \mathrm{M}$ of tbOH for $2 \mathrm{~h}$ or UVB irradiation $\left(100 \mathrm{~mJ} / \mathrm{cm}^{2}\right)$. After $24 \mathrm{~h}$ of serum starvation, the CM or control medium was added to the cells and incubated for $48 \mathrm{~h}$. The levels of elastin were estimated in culture supernatant by ELISA method. Collagen levels were estimated in cells by staining with Sirius red dye (Biocolor, UK).

\subsection{Measurement of Reactive 0xygen Species (ROS)}

Two thousand HFF-1 cells were seeded per well in a 96 well micro titer plate. After cell attachment, they were serum starved for 24 hours. The cells were then irradiated with UV-B $\left(100 \mathrm{~mJ} / \mathrm{cm}^{2}\right)$. The photo-damaged cells were immediately treated with varying concentrations of CM or control medium for $24 \mathrm{~h}$. Next, the cells were washed with $1 \times$ PBS (GIBCO, Carlsbad, USA) and incubated with 2',7'-dichlorofluorescin diacetate (DCFDA) dye (Abcam, Cambridge, UK), to measure ROS activity within the cells, for 30 minutes and the fluorescent readings were obtained.

\subsection{Cyclin B1 Estimation}

Two hundred fifty thousand HFF-1 cells were seeded per well in a 6 well plate 
and serum starved for $24 \mathrm{~h}$. After serum starvation, cells were treated with 300 $\mu \mathrm{M}$ of tbOH for $2 \mathrm{~h}$ following which the cells were grown in CM or control medium for $48 \mathrm{~h}$. The cell lysates were assayed by ELISA for Cyclin B1 (USCN life science, TX, USA).

\subsection{Statistical Analysis}

The data were analyzed using Graph Pad Prism 5 software (Graph Pad Software, Inc., La Jolla, CA, http://www.graphpad.com). Pairs of data sets were analyzed for statistical significance using Student's t test (95\% Confidence interval). P < 0.05 was considered significant. The results were presented as means \pm SEM. SEMs were shown as error bars in figures.

\section{Results}

\subsection{Evaluation of the CM Reveals the Presence of a Wide Variety of GF/CKs}

GF/CKs analyzed by the antibody array revealed a highly diverse composition of the CM in terms of various pleiotropic GF/CKs related to proliferation and migration of cell types belonging to different lineages, angiogenesis, epithelialization and wound healing, hematopoiesis and neural stimulation. Six different preparations of CM were run using this array and the results clearly show that these factors were present consistently and reproducibly in the preparations.

Among the factors involved in cellular proliferation, we detected a number of fibroblast growth factor family proteins such as FGF-4, FGF-6 and FGF-7/KGF, but not FGF-2, which are known to induce fibroblast and keratinocyte proliferation. Similarly, EGF, TGF $\alpha$, PDGF-AA, PDGF-AB and PLGF were also detected which are known to promote epithelial cell proliferation and migration. Additionally, angiogenic factors such as VEGF, PLGF, IGF-1 and HGF were also detected. A number of IGFBP's were also present in the CM that are known for inducing chemotaxis and proliferation of different cell types. Since the CM was derived from BMMSC, a number of growth factors like SCF, G-CSF, M-CSF, GM-CSF, which are known to play a role in hematopoiesis, were also found in the CM. Curiously, a number of neurotrophic factors such as NT-3, NT-4, NGF and GDNF involved in survival and proliferation of different types of neural cells were also found to be present in the CM. These apart, the presence of corresponding soluble receptors for some of these growth factors like VEGFR2, 3, EGF-R, PDGFRa, PDGFRb, SCF-R, M-CSFR and IGF-IIR were observed. With the exception of bFGF, all 40 factors were detected in the BMMSC CM, suggesting that $\mathrm{CM}$ is highly enriched in numerous GFs and cell signaling molecules that are intricately associated with cellular and tissue homeostasis (Figure 2).

Since the aim of the study was to determine the effect of BMMSC CM in improving and reversing the signs of skin aging, we selected 13 GFs and estimated their quantity in the CM. The GFs were chosen based on literature search and the activity ranged from cell proliferation, migration, angiogenesis and epithelialization, all of which are important steps for skin regeneration. We also selected 


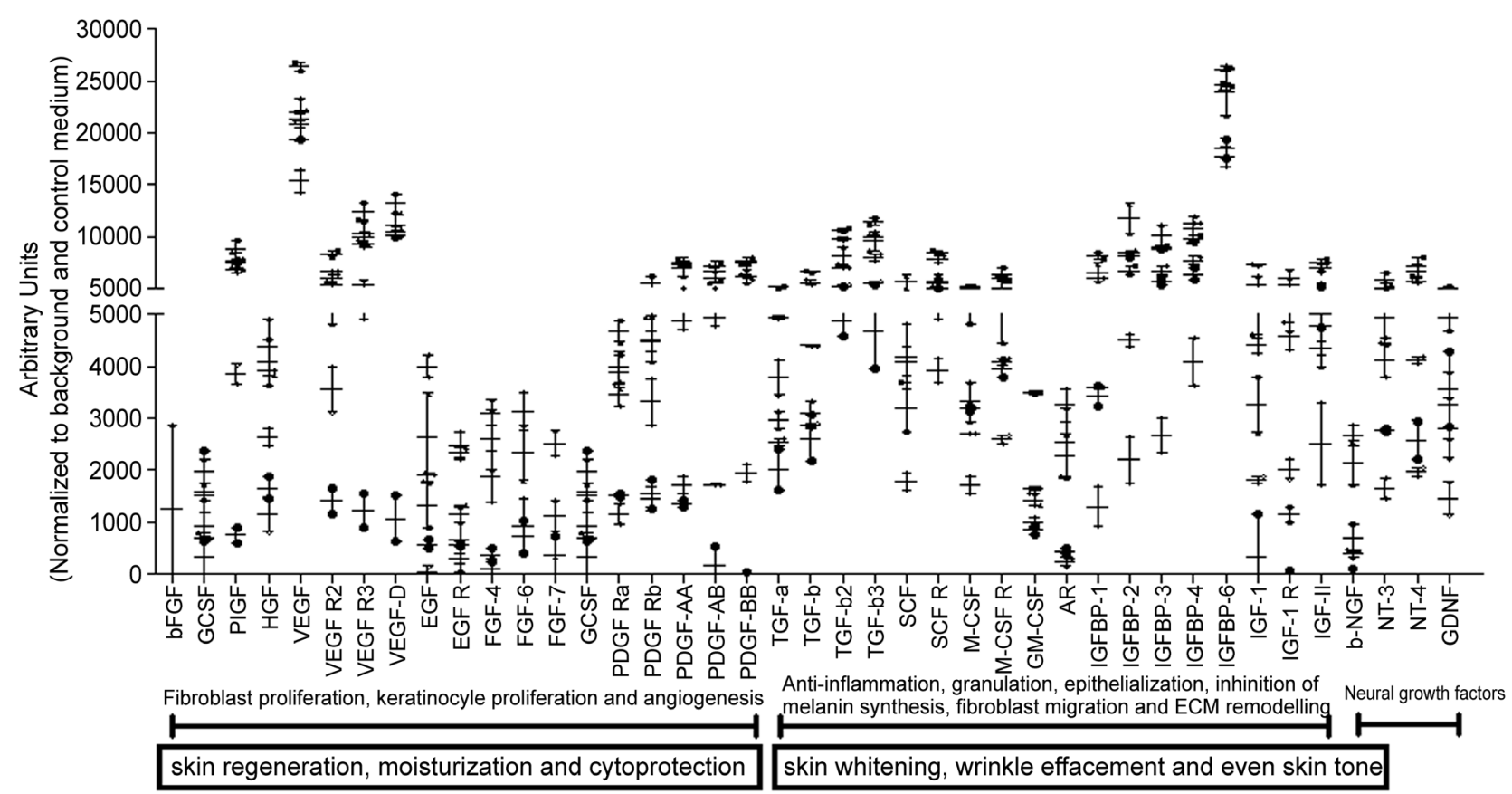

Figure 2. Secretome analysis of BMMSC-derived CM: Forty-one growth factor array analysis of six batches of BMMSC CM. The values are represented in arbitrary units, normalized to the background and control medium. Data is represented as mean \pm SEM.

a few factors such as Laminin - an extracellular matrix protein, tissue inhibitors of metalloproteinases, TIMP-1 and TIMP-2, which are known to inhibit ECM degradation by matrix metalloproteases (MMPs) and a pro-inflammatory marker-TNF- $\alpha$ (Figure 3 ). It is evident that while majority of factors responsible for fibroblast proliferation (FGF-7/KGF, PDGF), angiogenesis (VEGF, HGF, IGF-1, Ang-1), cell migration (SDF-1 $\alpha, \mathrm{M}-\mathrm{CSF}$ ) and epithelialization (TGF $\beta 1$, FGF-7/ KGF, Laminin, TIMP-1 and TIMP-2) are present in varying amounts ranging from $53 \mathrm{pg} / \mathrm{ml}$ to $2218000 \mathrm{pg} / \mathrm{ml}$. It is important to note that the systemic inflammatory marker TNF- $\alpha$ is present in the CM at a very negligible amount $(3.5 \mathrm{pg} / \mathrm{ml})$ and lowest among all the factors quantified. In addition to these, we also quantified the amount of PGE-2 $(230 \pm 80 \mathrm{pg} / \mathrm{ml})$ in three preparations of CM, which is a well reported molecule for anti-inflammatory function. These results prompted us to perform the next round of experiments to assess the effect of $\mathrm{CM}$ in a number of in vitro assays that are geared towards determining a cascade of cellular properties known to enhance skin aging.

\subsection{BMMSC Derived CM Treatment Promotes Proliferation and Migration of Dermal Fibroblasts and Increases the Synthesis of Elastin}

Proliferation and migration of dermal fibroblasts are important steps in the process of skin regeneration and repair. We used human foreskin fibroblast (HFF-1) line as an in vitro system to evaluate the proliferative effect and migratory potential of the BMMSC CM. As an initial proof of concept, we tested the $1 \times \mathrm{CM}$ unconcentrated and $10 \times$ concentrated CM on HFF-1 cells at different dilutions, alongside appropriate controls. We observed that BMMSC CM promoted the 


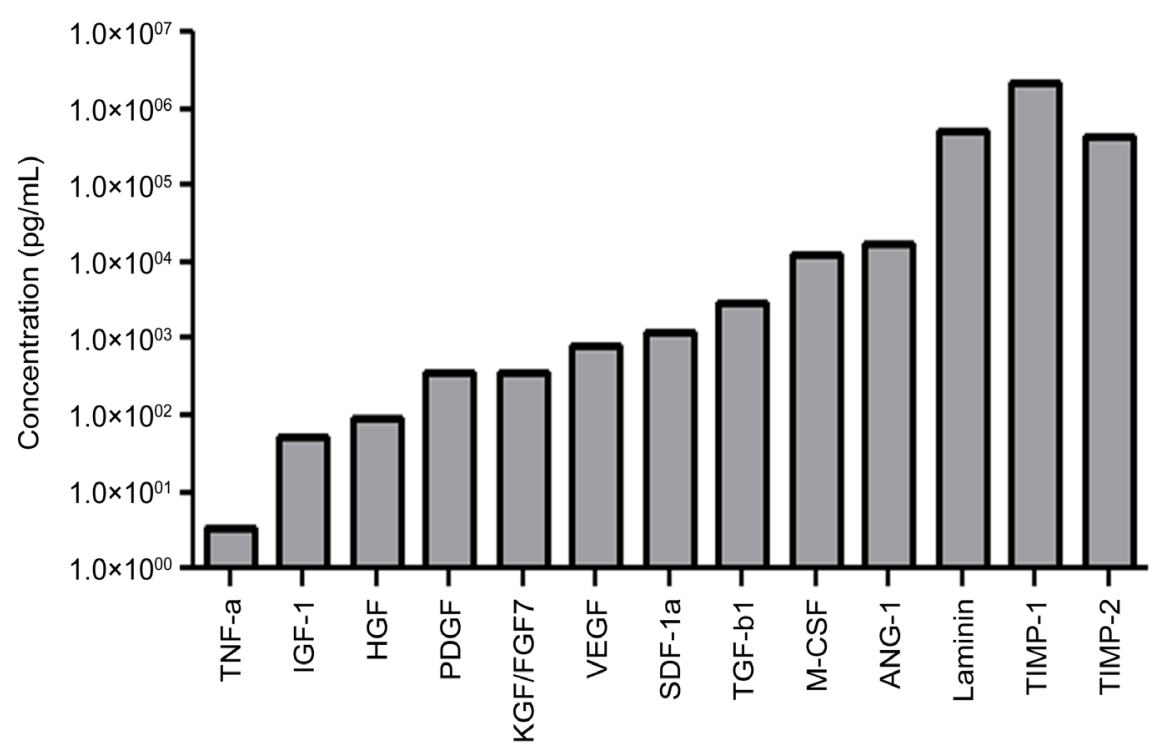

Figure 3. Quantification of GF/CKs in BMMSC-derived CM: Quantification of 13 selected GF/CKs by ELISA or Luminex multiplexing technology. The concentrations of the $\mathrm{GF} / \mathrm{CKs}$ are represented in $\mathrm{pg} / \mathrm{mL}$.

proliferation of HFF starting from a dilution of 5\%, although significant differences in proliferation potential between the $\mathrm{CM}$ and control medium were observed at $25 \%(\mathrm{P} \leq 0.02)$ and $50 \%(\mathrm{P} \leq 0.0091)$ dilution. The proliferation effect of the CM on HFF cells was saturated at $100 \%$ concentration (Figure $4(\mathrm{~A})$ ). We also observed that $10 \times$ BMMSC CM significantly promoted HFF proliferation at $25 \%(\mathrm{P} \leq 0.002)$ and $50 \%(\mathrm{P} \leq 0.002)$ dilution compared to the $10 \times$ control medium although not a magnitude of the unconcentrated $1 \times \mathrm{CM}$ at the same dilutions. Undiluted $10 \times \mathrm{CM}$ did not promote the proliferation of HFF cells, perhaps due to excessive protein load and lack of other nutrients. No HFF proliferation was detected in the serum free medium which was used as negative control (Figure 4(A), Figure 4(B)).

The effect on migration of HFF-1 cells using BMMSC CM and 10× CM was evaluated at $5 \%, 10 \%, 25 \%$ and $50 \%$ dilutions, by a scratch wound assay. The $\mathrm{CM}$ promoted greater than $80 \%$ of migration of HFF into the scratched area beginning from the $5 \%$ of $\mathrm{CM}$ dilution, indicating that low concentrations of growth factors were sufficient to promote migration of HFF. Enhanced migration was observed in the CM group as compared to the control at 5\% ( $\mathrm{P} \leq 0.03), 10 \%(\mathrm{P} \leq$ $0.0004), 25 \%(\mathrm{P} \leq 0.02)$ and $50 \%(\mathrm{P} \leq 0.02)$ dilution, suggesting the superior effect was caused by human GF/CKs secreted by BMMSC. No significant differences in the migration promoting capacity were observed between the undiluted $\mathrm{CM}$ and control, indicating the saturation limit for the effect of the human growth factors promoting the migration of HFF (Figure $4(\mathrm{C})$ ). Similar migratory capacity of the $10 \times \mathrm{CM}$ was observed where significant migration was observed at $5 \%(\mathrm{P} \leq 0.0085), 10 \%(\mathrm{P} \leq 0.02)$ and $25 \%(\mathrm{P} \leq 0.0001)$ dilution. The migration was higher in the $10 \times \mathrm{CM}$ at $50 \%$ dilution compared to the control, although not significant. The undiluted CM did not promote the migration of 
(A)

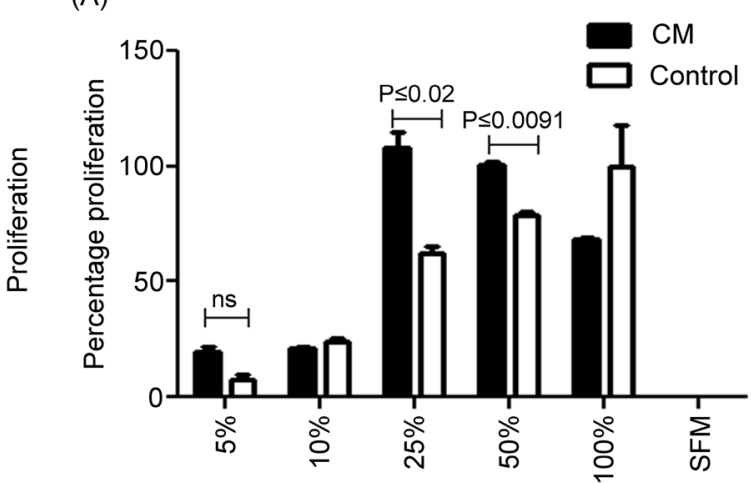

(B)

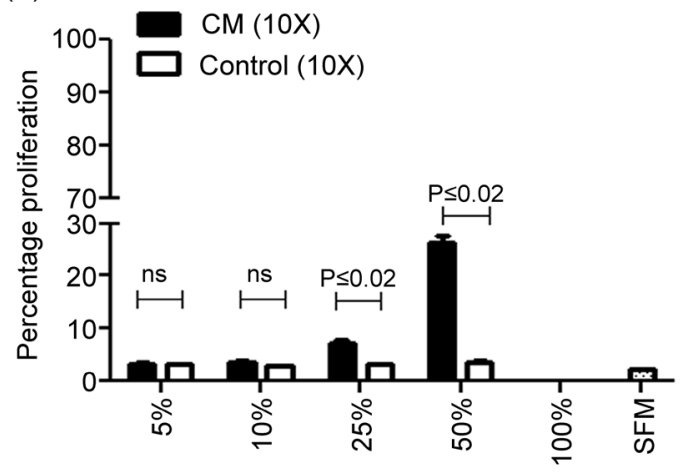

(C)

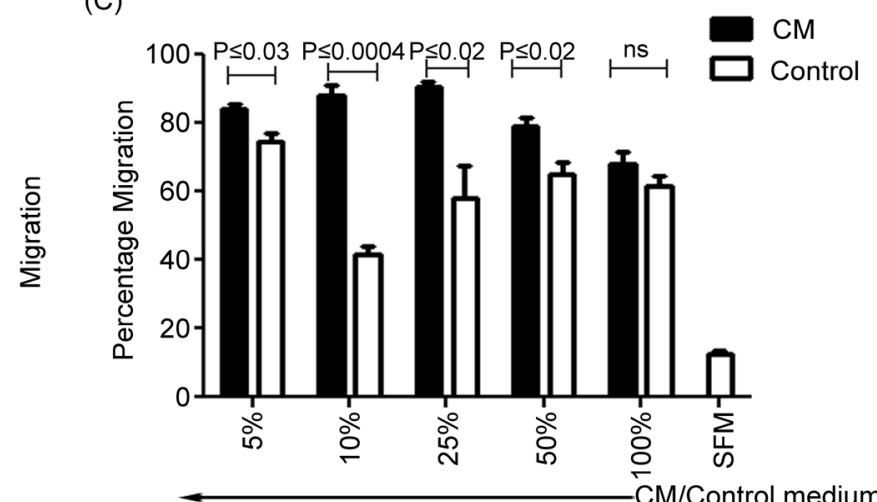

(D)

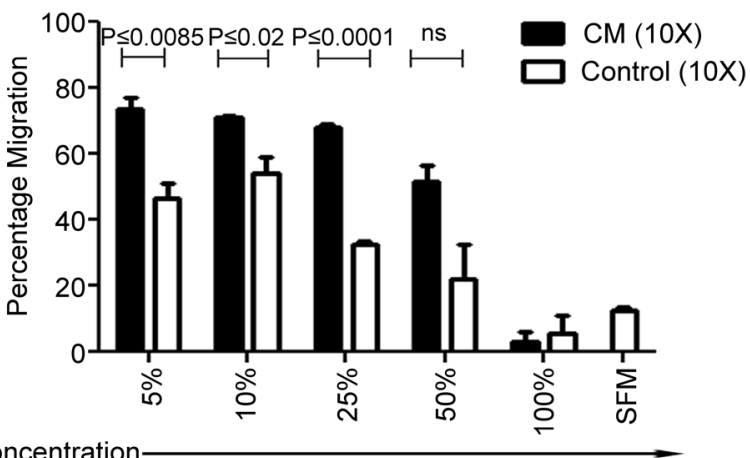

(E)

Elastin basal

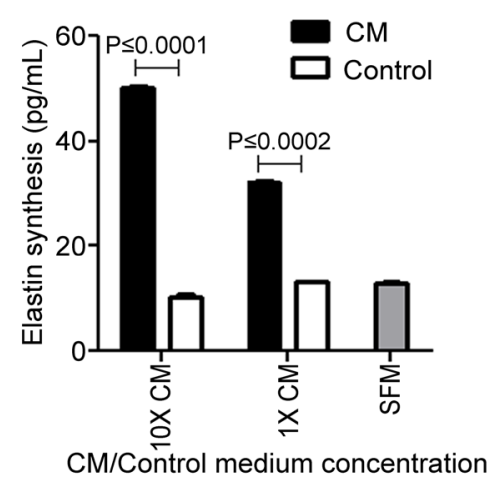

Figure 4. In vitro functional assays using HFF: (A) Proliferation of HFF using $1 \times$ BMMSC CM and $1 \times$ control medium; (B) Proliferation of HFF using 10× BMMSC CM and 10× control medium; (C) Migration of HFF using $1 \times$ BMMSC CM and $1 \times$ control medium; (D) Migration of HFF using 10× BMMSC CM and 10× control medium; (E) Estimation of basal elastin synthesisby HFF using $1 \times$ and $10 \times \mathrm{CM}$ and respective control mediums. Serum free medium (SFM) was used as the negative control. Data is represented as mean \pm SEM.

HFF similar to that of proliferation, seemingly due to the high concentrations of protein present in the $10 \times \mathrm{CM}$. Less than $20 \%$ migration was promoted by the serum free medium control (Figure 4(D)).

In addition to proliferation and migration of the dermal fibroblasts, we also estimated the level of elastin on the dermal fibroblasts, pretreated with the CM. Treatment of HFF-1 cells with $1 \times \mathrm{CM}$ for $72 \mathrm{~h}$ led to increase in elastin levels. The increase was measured at $146 \%$ and $368 \%$ for $1 \times \mathrm{CM}$ and $10 \times \mathrm{CM}$ when 
compared against their respective $1 \times$ or $10 \times$ control medium (Figure $4(\mathrm{E})$ ). This data clearly suggests that BMMSC CM may possess the ability to promote skin tightening and restore skin elasticity.

\subsection{Protection of BMMSC-Derived CM against Oxidative Damage}

We evaluated the anti-aging potential of BMMSC-derived CM through a series of in vitro assays. First, we looked into the cytoprotective/viability restorative effects of $10 \times \mathrm{CM}$ against oxidative damage in dermal fibroblasts, post treatment with $300 \mu \mathrm{M}$ of tbOH for $2 \mathrm{~h}$ and post treatment of damaged fibroblast with $10 \times$ CM for $48 \mathrm{~h}$ was evaluated by MTT assay. Overall, we observed that treatment of HFF-1 cells with $300 \mu \mathrm{M}$ of tbOH led to a $45 \%$ decrease in cellular viability in SFM treated group as compared to untreated cells. The cytoprotection obtained with $10 \times \mathrm{CM}$ was $11 \%, 18 \%$ and $12 \%$ higher compared to the $10 \times$ control medium at concentrations of $5 \%$ ( $76 \%$ cytoprotection), $10 \%$ ( $73 \%$ cytoprotection) and $50 \%$ ( $75 \%$ cytoprotection). The above result indicates that GF/CKs in the lowest concentration of $5 \%$ and $10 \% 10 \times \mathrm{CM}(\mathrm{P} \leq 0.04)$, was sufficient to protect HFF cells against tbOH induced oxidative stress (Figure 5(A-a)).

Oxidative stress is known to damage ECM components, so we evaluated the protective/restorative effects of $10 \times \mathrm{CM}$ and $10 \times$ control medium against collagen,

(A) VIABILTY

(a)

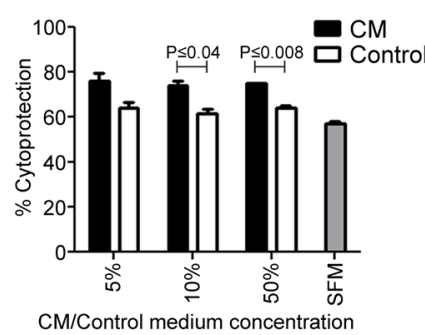

(B) PROTECTION OF EXTRACELLULAR MATRIX COMPONENTS

(a)

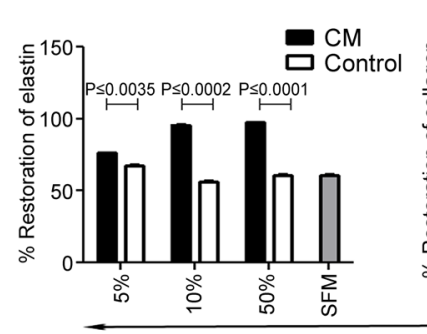

(b)

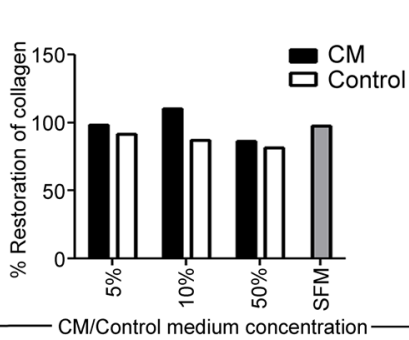

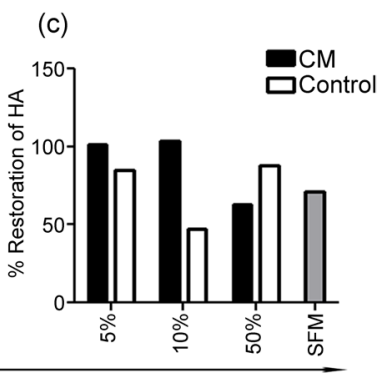

(C)

(a)

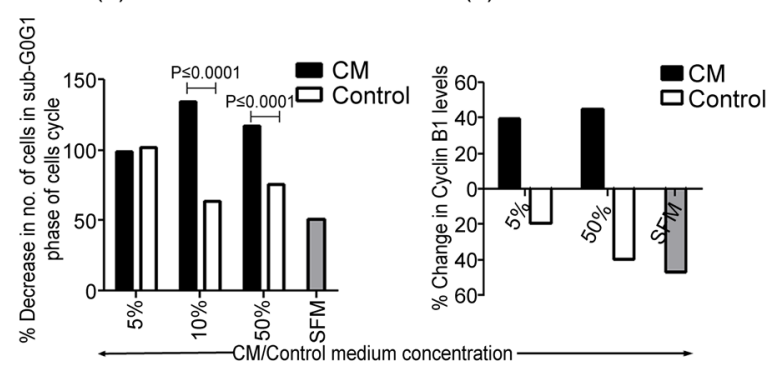

Figure 5. Effects of BMMSC CM in reducing oxidative stress in HFF: (A-a) Percentage viability or cytoprotection induced by 10× BMMSC CM and 10× control medium, at different concentration post tbOH induced oxidative stress on HFF; (B) Protection of ECM components by $10 \times$ BMMSC CM and 10× control mediumat different concentrations-(B-a) Percentage restoration of elastin, (B-b) Percentage restoration of collagen, (B-c) Percentage restoration of hyaluronic acid (HA), in HFF after tbOH induced oxidative stress; (C) Anti-senescence activity in HFF cells after tbOH induced oxidative stress followed by $10 \times \mathrm{BMMSC}$ CM and $10 \times$ control medium treatmentusing different concentrations, (C-a) Percentage decrease in the number of cells in sub G0/G1 phase of the cell cycle, (C-b) Percentage increase in the cyclin B1 levels. Data is represented as mean \pm SEM. 
elastin and hyaluronic acid degradation caused by oxidative damage in dermal fibroblast cells after 48 hours of treatment of tbOH. Treatment of tbOH damaged HFF-1 cells with $10 \times \mathrm{CM}$ led to $9 \%, 39 \%$ and $36 \%$ greater restoration of elastin levels at 5\% (P $\leq 0.0035), 10 \%(\mathrm{P} \leq 0.0002)$ and $50 \%(\mathrm{P} \leq 0.0001)$ concentrations with respect to $10 \times$ control medium treated cells upon tbOH treatment (Figure 5(B-a)). Similarly, 10× CM was able to restore 7\%, 23\% and $6 \%$ of collagen levels higher compared to respective $10 \times$ control medium at 5\%,10\% and $50 \%$ concentrations, although no significance was perceived between the $10 \times \mathrm{CM}$ treated and their respective control medium groups, we observed enhanced collagen synthesis in the $10 \%$ concentration indicating a greater contribution of human GF/CKs at this particular concentration (Figure 5(B-b)).

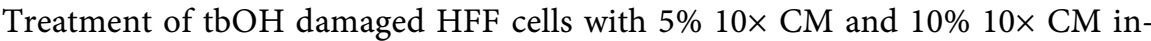
creased hyaluronic acid levels by $17 \%$ and $55 \%$ respectively, again indicated that $10 \%$ concentration of the $10 \times \mathrm{CM}$ elicits the best result and a higher concentration of the CM may have a saturating effect (Figure 5(B-c)).

The anti-apoptotic effects of the 10x CM against oxidative stress-induced apoptosis in dermal fibroblast cells after tbOH treatment at $300 \mu \mathrm{M}$ for $2 \mathrm{~h}$, were evaluated by analyzing the percentage of cells in the sub-G0/G1 phase of the cell cycle after $48 \mathrm{~h}$ of damage. Post-treatment of $10 \times \mathrm{CM}$ led to $71 \%$ and $41 \%$ decrease in a number of cells in the sub-G0/G1 phase of the cell cycle at doses of $10 \%(\mathrm{P} \leq 0.0001)$ and $50 \%(\mathrm{P} \leq 0.0001)$ as compared to $10 \times$ control medium treated cells respectively. However, at a lower dose of $5 \%$ of the $10 \times \mathrm{CM}$ dilution, no difference in the number of the sub G0/G1 cells was observed suggesting that the most effective anti-senescence activity at $10 \%$ dilution of $10 \times \mathrm{CM}$ (Figure 5(C-a)). These data together demonstrate enhanced anti-aging effects at $5 \%$ and $10 \%$ dilutions of $10 \times$ BMMSC CM.

\subsection{Protection of BMMSC-Derived CM against UV-B Damage}

Protective effect of the CM against the UV-B induced damage was also assessed by series of in vitro assays in the identical manner as was with tbOH induced oxidative stress studies. First, we looked into the restorative effect on the viability of the cells HFF cells following the exposure to UVB radiation $\left(230 \mathrm{~mJ} / \mathrm{cm}^{2}\right)$. Viability of HFF cells was analyzed by MTT assay 48 hours after UV damage. Treatment with $10 \times \mathrm{CM}$ led to $18 \%, 8 \%$ and $26 \%$ greater cytoprotective effects in photo-damaged HFF-1 cells at the dilutions of 5\% $10 \%$ and $50 \%$ respectively as compared to respective $10 \times$ control medium treated cells (Figure $6(\mathrm{~A}-\mathrm{a})$ ).

Subsequently, we evaluated the restorative effect of the CM on ECM components, elastin, collagen and hyaluronic acid at the end of 48 hours in photo-damaged HFF cells. The percentage restoration of elastin with $10 \times \mathrm{CM}$ treatments in comparison to $10 \times$ control medium was observed to be $120.2 \%$ and $50.1 \%$ at dilutions of $10 \%$ and $50 \%$ respectively indicating the maximum contribution of human GF/CKs in $10 \%$ of $10 \times \mathrm{CM}$ in restoring the elastin levels. Restorative effects of the $\mathrm{CM}$ at $5 \%$ dilution as not observed in comparison to the $5 \%$ diluted control medium (Figure 6(B-a)). Percentage restoration of collagen 


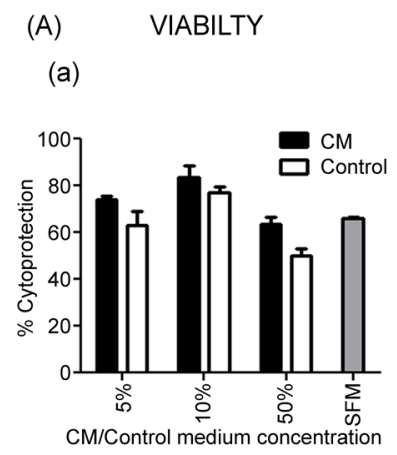

(B)

(a)

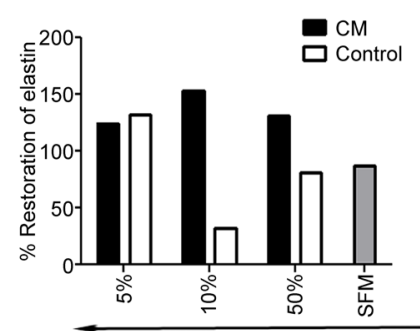

(C)
PROTECTION OF EXTRACELLULAR MATRIX COMPONENTS

(b)

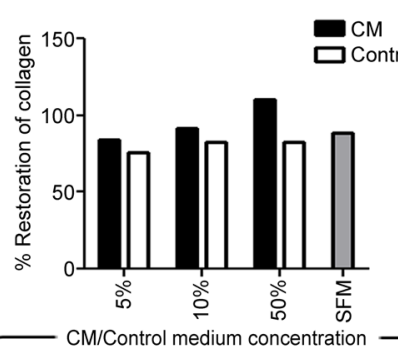

(c)

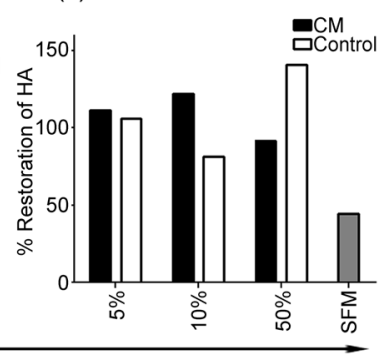

(a)

ANTI-SENESCENCE

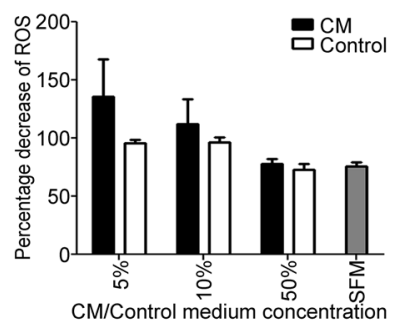

Figure 6. Effects of BMMSC CM in reducing UV-B induced damage in HFF: (A-a) Percentage viability or cytoprotection induced by $10 \times$ BMMSC CM and 10× control medium at different concentrations after UV-B damage to HFF; (B) Protection of ECM components by $10 \times$ BMMSC CM and 10× control medium at different concentrations-(B-a) Percentage restoration of elastin, (B-b) Percentage restoration of collagen, (B-c) Percentage restoration of hyaluronic acid (HA), in HFF after UV-B damage; (C) Anti-senescence activity in HFF post damage by UV-B, followed by $10 \times$ BMMSC CM and 10× control medium treatment at different concentrations-(C-a) Percentage decrease in ROS levels. Data is represented as mean \pm SEM.

was $8.4 \%, 8.8 \%$ and $28.1 \%$ higher in the $10 \times \mathrm{CM}$ treated group at the dilutions of $5 \%, 10 \%$ and $50 \%$ respectively compared to respective $10 \times$ control medium dilutions, suggesting that human GF/CKs contributed to collagen synthesis in a dose dependent manner (Figure 6(B-b)). Similar to elastin and collagen restoration, hyaluronic acid synthesis at $5 \%$ and $10 \%$ dilution of $10 \times \mathrm{CM}$ was observed to be $5.5 \%$ and $40.8 \%$ higher compared to respective $10 \times$ control medium dilutions further indicating $10 \%$ dilution of the $10 \times \mathrm{CM}$ possessing superlative restorative effects. At $50 \%$ dilution of $10 \times \mathrm{CM}$, hyaluronic acid restoration was stunted compared to its $10 \times$ control medium possibly due to saturating concentrations of proteins in the CM (Figure $6(\mathrm{~B}-\mathrm{C})$ ). Overall, $10 \%$ dilution of BMMSC $10 \times$ $\mathrm{CM}$ treatment led to improved restoration of key structural proteins like collagen, elastin and hyaluronic acid on the photodamaged fibroblasts.

UVB irradiation is known to increase the level of reactive oxygen species (ROS) accumulation in living cells that trigger the activation of the mitochondrial-mediated apoptotic pathway [20]. The effect of 10× CM against UV-B induced ROS generation in photo-damaged fibroblasts following was evaluated by measuring the amount of oxidized DCFDA dye $48 \mathrm{~h}$ after UV-B irradiation. Treatment of $10 \times \mathrm{CM}$ at dilutions of $5 \%, 10 \%$ and $50 \%$, reduced the levels of ROS by $38.3 \%, 17.5 \%$ and $5.8 \%$ compared to the respective dilutions of $10 \times$ control medium, whereas for control medium. This result clearly indicates the contribution of human GF/CKs in reducing the levels ROS most effectively at 5\% 
and $10 \%$ dilutions of the $10 \times \mathrm{CM}$ (Figure $6(\mathrm{C}-\mathrm{a}))$.

\section{Discussion}

The extensive amount of basic, preclinical and clinical research performed over the past decades has clearly demonstrated that stem cells derived from many tissues intrinsically possess potential for tissue repair and regeneration. Multipotent MSCs from both adult and neonatal tissues have been shown to initiate and sustain tissue repair when these cells are delivered either by systemic or local administration [21] [22]. Initially, it was thought that the reparative effect of MSC was due to their differentiation ability in situ to specific cell types that were lost or damaged due to many reasons like aging, injury, environmental insults etc. [11]. Subsequent studies have demonstrated that the MSCs are capable to secrete a wide variety of trophic factors that comprise the "proteome" or "secretome" of these cells and may be causally related for the therapeutic effect observed by these cells [16] [23] [24] [25]. Literature evidence suggest that MSCs can secrete over 200 different types of factors that encompasses a large array of growth factors, cytokines and chemokines, and extracellular matrix proteins and enzymes, many of which may work in conjunction or independently and essentially trigger tissue repair and possibly rejuvenation [20] [26] [27].

The great majority of MSC-based therapeutic technology requires large scale expansion and, in the process, generates high volume of conditioned or spent medium enriched in bioactive factors. The secretome signature of MSC has been reported to vary between human donors, which may lead to considerable difference in the CM composition [12]. To minimize such variability, we used a three donor pool of BMMSC and expanded these cells to generate the CM. We have presented here the partial composition of the secretome of the pooled BMMSC derived $\mathrm{CM}$ and demonstrated reproducibility of the method used, and consistency of the CM composition. Furthermore, using a variety of in vitro cell based assays; we show that the CM was able to induce proliferation, migration, ECM synthesis etc., in fibroblasts damaged by extraneous elements, thus suggesting that it may be useful for skin repair and rejuvenation.

Aging of the skin both externally and internally is accelerated by continuous exposure to environmental toxins as well as by the sun light and can ultimately lead to reduction in skin firmness and wrinkle formation. Despite the tremendous advancement made in the field of cosmetic product market using small molecules, biopolymers, crude and purified plant extracts, available market research data suggest that there is still a great opportunity for aggressive product development for skin health sector. The bioactive factors secreted by MSC may eventually fill this niche because of their potential to repair skin damage and aid in skin rejuvenation. Amongst the latest developments in the cosmetic industry, use of biotechnology-derived ingredients, nutritional regimens, fibroblast or stem cell-based products are becoming increasingly popular, particularly for the aging population [13]. The unique characteristics of MSCs unraveled over the past decades, are not only being exploited for the rapidly growing field of rege- 
nerative medicine, but also provide a promising platform for generation of novel cosmetics. In fact, paracrine factors such as PDGF, bFGF, KGF, TGF $\beta 1$, HGF, VEGF, Type-1 collagen and fibronectin secreted by adipose-derived mesenchymal stem cells (ADSC) have been shown to restore dermal thickness, improve skin texture and reduce wrinkles in human skin [14].

GF/CKs are important regulatory molecules which mediate the signaling pathways between and within the cells and play an important role in maintaining the skin structure and function [15]. BMMSC CM contains factors which are anti-apoptotic-like IGF-1, GM-CSF, MCSF, and SCF; angiogenic factors like VEGF, PLGF, Ang-1 and HGF; anti-inflammatory factors such as HGF and TGF $\beta 1$, and also factors that promote proliferation and migration of progenitor cells like FGF-4, SDF-1, IGF-1, PDGF and GM-CSF. These cytokines and growth factors as well as other molecules like PGE-2 secreted by BMMSCs are known to down regulate inflammation following injury or damage due to external insults like continuous exposure to radiation, or laser treatment [16].

Dermal fibroblasts are important for ECM productions as well as for interacting with keratinocytes for both normal maintenance as well after radiation-induced damage of the skin. This cross talk between the fibroblasts and keratinocytes involves proliferation and DNA repair of the latter cell type [28] [29]. In this study, we have demonstrated anti-aging and anti-wrinkling potential of BMMSC derived CM using a comprehensive multiparametric analysis by inducing oxidative stress and UV-B damage in dermal fibroblast system in vitro. Our results clearly show that the CM exhibited multiple beneficial effects on the damaged fibroblasts. Whether the CM used in this study would also exert similar effects on keratinocytes was beyond the scope of this investigation. However, based on the profile of the $\mathrm{CM}$ and its positive effects on dermal fibroblasts, it is tempting to speculate that it may similar effect on keratinocytes damaged by oxidative stress or UV-B. Firstly, treatment with CM or 10× CM led to enhancement of fibroblast proliferation and migration as compared to cells treated with SFM. Also, treatment with CM or $10 \times \mathrm{CM}$ led to considerable increase in synthesis of basal levels of extracellular matrix component like elastin which is a key protein responsible for skin tightening and retention of skin elasticity. In a study published earlier, it has been documented that CM from adipose tissue has a similar effect on dermal fibroblast [17]. In addition, our BMMSC CM was able to restore the viability and ECM integrity following the induction of oxidative stress and UV-B damage. Similarly, the anti-senescence effect of the CM has been demonstrated by an increase in the cyclin B1 levels against oxidative stress induced dermal fibroblast. Cyclin B1 is a regulatory protein whose level decrease in the cells undergoing senescence. BMMSC CM has also rendered protection against UV-B induced photo-damage on the dermal fibroblast by decreasing the ROS generation. The ROS generated, contributes to UV-induced skin damage, leading to premature aging and cancer [6] [30] but, the exact mechanisms through which MSCs offer protection has not been evaluated in this study. Taking all these in vitro data together BMMSC CM possess all the pre requisite properties for skin regeneration, protection against skin damage by oxidative 
stress and UVB damage and maintain the integrity of skin structure.

The results obtained in this study corroborates with the data published by Kwon et al., where they have demonstrated the anti-wrinkling potential of human BMMSC CM by inducing fibroblast proliferation and pro-collagen synthesis [18]. CM from adipose tissue comprising of several growth factors and cytokines has also shown to demonstrate the similar effects in fibroblasts and keratinocytes [31] [32]. To further illustrate the plausible function of some of the key factors present in our CM, a schematic diagram is shown in Figure 7. Taking all this data together BMMSC derived CM could be a rich source for developing anti-aging and anti-wrinkling cosmetic products.

\section{Conclusion}

In summary, using the CM obtained from a pooled population of human BMMSC, we show that it could actively modulate various biological and biochemical pathways in cells following exposure to stress inducing factors. Based on the results presented here, our next aim is to develop a skin care formulation with this $\mathrm{CM}$ for accelerating repair of damaged skin and stimulate skin rejuvenation.

\section{Acknowledgements}

We thank Stempeutics Research Pvt Ltd for funding this study, Ms. Devi Da-
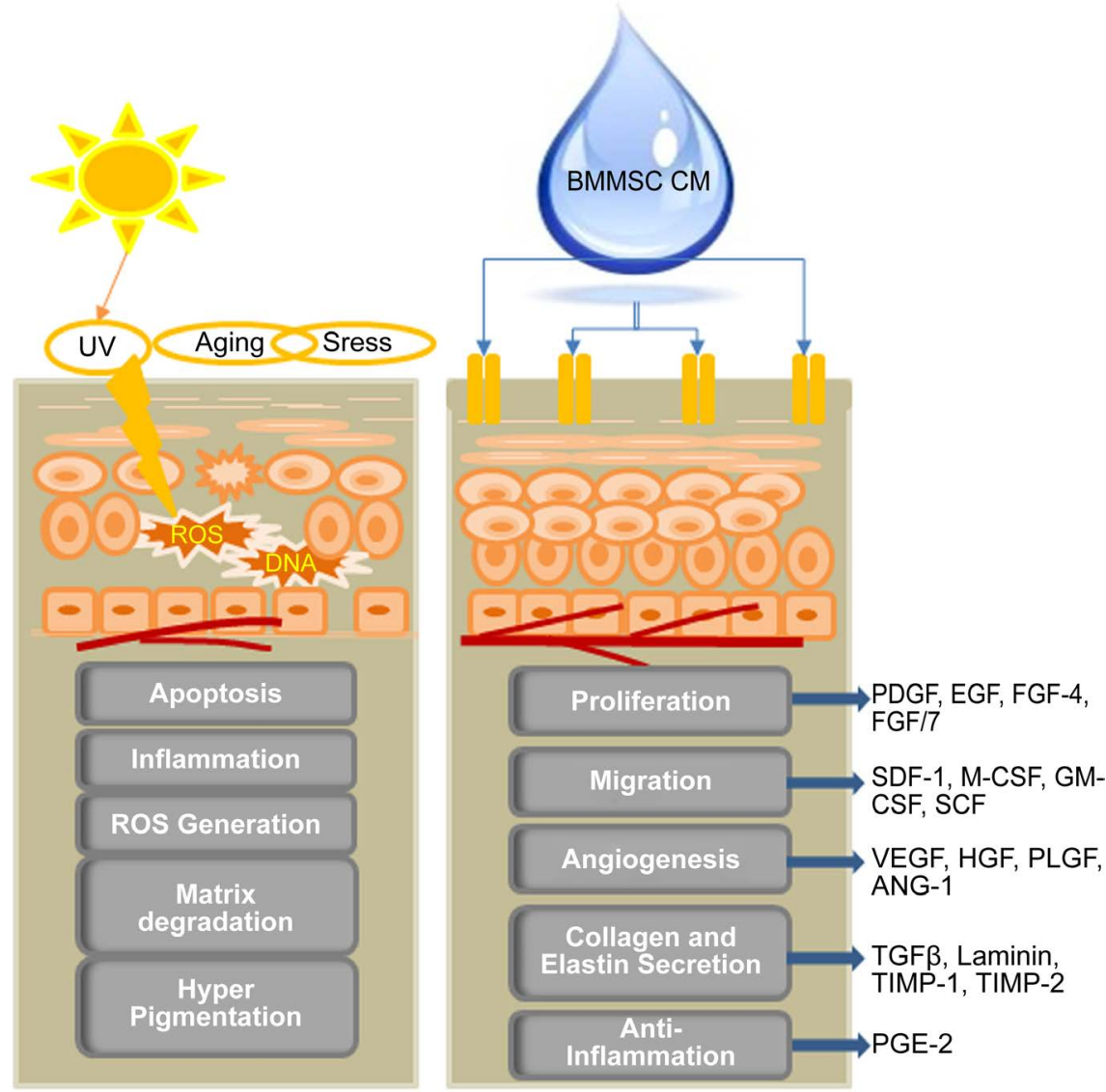

Figure 7. Proposed mechanism of action of BMMSC-derived CM for skin rejuvenation. 
modaran and Mr. Pachaiyappan Vishwanathan of the production and quality control teams for providing the cells and concentrating the medium, Dr. Muthukumar Amirthalingam for reviewing the manuscript and Srijith Kadungolath for helping us with the graphical presentation of the figures and formatting the manuscript. We also thank Syngene International Ltd. and Dabur Research Foundation for supporting as CRO's.

\section{Ethical Approval}

All bone marrow aspirates were obtained with written informed consent using the guidelines approved by the Institutional Committee for Stem Cell Research (IC-SCR) and the Institutional Ethics Committee (IEC) at the Kasturba Medical College, Manipal, Karnataka, India. The study was also approved by both the committees.

\section{Consent for Publication}

All participating authors consent the publication of this article.

\section{Competing Interests}

All participating authors hereby declare no competing interests.

\section{Funding}

The study was funded by Stempeutics Research Pvt. Ltd., Bangalore, India.

\section{Author Contributions}

S.B and C.T.: conception and design, collection and/or assembly of data, data analysis and interpretation, manuscript writing; A.W.: collection and/or assembly of data; P.S.: collection and/or assembly of data; P.K.G.: data analysis and interpretation; R.N.S.: data analysis and interpretation; A.S.M.: conception and design, data analysis and interpretation, final approval of manuscript. All authors read and approved the manuscript.

\section{References}

[1] Ehrlich, M., Rao, J., Pabby, A. and Goldman, M.P. (2006) Improvement in the Appearance of Wrinkles with Topical Transforming Growth Factor $\beta 1$ and L-Ascorbic Acid. Dermatologic Surgery, 32, 618-625.

[2] Gold, M.H., Goldman, M.P. and Biron, J. (2007) Efficacy of Novel Skin Cream Containing Mixture of Human Growth Factors and Cytokines for Skin Rejuvenation. Journal of Drugs in Dermatology, 6, 197-201.

[3] Fitzpatrick, R. and Rostan, E. (2003) Reversal of Photodamage with Topical Growth Factors: A Pilot Study. Journal of Cosmetic and Laser Therapy, 5, 25-34. https://doi.org/10.1080/14764170310000817

[4] Sanderson, J. and Taylor, G. (2013) Scientific Rationale for Use of Cytokine-Based Topical Adjuvants in Aesthetic Medical Practice. Cellese Regenerative Therapeutics.

[5] Ganceviciene, R., Liakou, A.I., Theodoridis, A., et al. (2012) Skin Anti-Aging Strategies. Dermato-Endocrinology, 4, 308-319. https://doi.org/10.4161/derm.22804 
[6] Kim, W.-S., Park, B.-S. and Sung, J.-H. (2009) Protective Role of Adipose-Derived Stem Cells and Their Soluble Factors in Photoaging. Archives of Dermatological Research, 301, 329-336. https://doi.org/10.1007/s00403-009-0951-9

[7] Rahman, K. (2007) Studies on Free Radicals, Antioxidants, and Co-Factors. Clinical Interventions in Aging, 2, 219-236.

https://www.ncbi.nlm.nih.gov/pmc/articles/PMC2684512/

[8] Bernardo, M.E., Locatelli, F. and Fibbe, W.E. (2009) Mesenchymal Stromal Cells: A Novel Treatment Modality for Tissue Repair. Annals of the New York Academy of Sciences, 1176, 101-117. https://doi.org/10.1111/j.1749-6632.2009.04607.x

[9] Riccobono, D., François, S., Valente, M., et al. (2014) Advances in Stem Cell Therapy: Specific Applications in the Treatment of Cutaneous Radiation Syndrome. Journal of Stem Cell Research \& Therapy, 4, 186.

https://doi.org/10.4172/2157-7633.1000186

[10] Boomsma, R. and Geenen, D.L. (2012) Mesenchymal Stem Cells Secrete Multiple Cytokines That Promote Angiogenesis and Have Contrasting Effects on Chemotaxis and Apoptosis. PLoS ONE, 7, e35685. https://doi.org/10.1371/journal.pone.0035685

[11] Pal, R., Hanwate, M., Jan, M. and Totey, S. (2009) Phenotypic and Functional Comparison of Optimum Culture Conditions for Upscaling of Bone Marrow-Derived Mesenchymal Stem Cells. Journal of Tissue Engineering and Regenerative Medicine, 3, 163-174. https://doi.org/10.1002/term.143

[12] Gottipamula, S., Sharma, A., Krishnamurthy, S., et al. (2012) Human Platelet Lysate Is an Alternative to Fetal Bovine Serum for Large-Scale Expansion of Bone Marrow-Derived Mesenchymal Stromal Cells. Biotechnology Letters, 34, 1367-1374. https://doi.org/10.1007/s10529-012-0893-8

[13] Gottipamula, S., Ashwin, K.M., Muttigi, M.S., et al. (2014) Isolation, Expansion and Characterization of Bone Marrow-Derived Mesenchymal Stromal Cells in SerumFree Conditions. Cell and Tissue Research, 356, 123-135. https://doi.org/10.1007/s00441-013-1783-7

[14] Jayaraman, P., Nathan, P., Vasanthan, P., et al. (2013) Stem Cells Conditioned Medium: A New Approach to Skin Wound Healing Management. Cell Biology International, 37, 1122-1128. https://doi.org/10.1002/cbin.10138

[15] Kwon, T.-R., Oh, C.T., Choi, E.J., et al. (2016) Conditioned Medium from Human Bone Marrow-Derived Mesenchymal Stem Cells Promotes Skin Moisturization and Effacement of Wrinkles in UVB-Irradiated SKH-1 Hairless Mice. Photodermatology, Photoimmunology \& Photomedicine, 32, 120-128. https://doi.org/10.1111/phpp.12224

[16] Sundaram, H., Mehta, R.C., Norine, J.A., et al. (2009) Topically Applied Physiologically Balanced Growth Factors: A New Paradigm of Skin Rejuvenation. Journal of Drugs in Dermatology, 8, 4-13.

[17] Kuçi, Z., Bönig, H., Kreyenberg, H., et al. (2016) Mesenchymal Stromal Cells from Pooled Mononuclear Cells of Multiple Bone Marrow Donors as Rescue Therapy in Pediatric Severe Steroid-Refractory Graft-Versus-Host Disease: A Multicenter Survey. Haematologica, 101, 985-994. https://doi.org/10.3324/haematol.2015.140368

[18] Zhukareva, V., Obrocka, M., Houle, J.D., et al. (2010) Secretion Profile of Human Bone Marrow Stromal Cells: Donor Variability and Response to Inflammatory Stimuli. Cytokine, 50, 317-321. https://doi.org/10.1016/j.cyto.2010.01.004

[19] Mindaye, S.T., Ra, M., Lo Surdo, J.L., et al. (2013) Global Proteomic Signature of Undifferentiated Human Bone Marrow Stromal Cells: Evidence for Donor-to-Donor Proteome Heterogeneity. Stem Cell Research, 11, 793-805.

https://doi.org/10.1016/j.scr.2013.05.006 
[20] Ranganath, S.H., Levy, O., Inamdar, M.S. and Karp, J.M. (2012) Harnessing the Mesenchymal Stem Cell Secretome for the Treatment of Cardiovascular Disease. Cell Stem Cell, 10, 244-258. https://doi.org/10.1016/j.stem.2012.02.005

[21] Prockop, D.J. and Youn Oh, J. (2012) Mesenchymal Stem/Stromal Cells (MSCs): Role as Guardians of Inflammation. Molecular Therapy, 20, 14-20. https://doi.org/10.1038/mt.2011.211

[22] Jackson, W.M., Nesti, L.J. and Tuan, R.S. (2012) Mesenchymal Stem Cell Therapy for Attenuation of Scar Formation during Wound Healing. Stem Cell Research \& Therapy, 3, 20. https://doi.org/10.1186/scrt111

[23] da Silva Meirelles, L., Fontes, A.M., Covas, D.T. and Caplan, A.I. (2009) Mechanisms Involved in the Therapeutic Properties of Mesenchymal Stem Cells. Cytokine \& Growth Factor Reviews, 20, 419-427. https://doi.org/10.1016/j.cytogfr.2009.10.002

[24] Caplan, A.I. and Dennis, J.E. (2006) Mesenchymal Stem Cells as Trophic Mediators. Journal of Cellular Biochemistry, 98, 1076-1084. https://doi.org/10.1002/jcb.20886

[25] Chen, L., Tredget, E.E., Wu, P.Y.G., et al. (2008) Paracrine Factors of Mesenchymal Stem Cells Recruit Macrophages and Endothelial Lineage Cells and Enhance Wound Healing. PLoS ONE, 3, e1886. https://doi.org/10.1371/journal.pone.0001886

[26] Flower, T.R., Pulsipher, V. and Moreno, A. (2015) A New Tool in Regenerative Medicine: Mesenchymal Stem Cell Secretome. Journal of Stem Cell Research \& Therapy, 1, 10-12. https://doi.org/10.15406/jsrt.2015.01.00005

[27] Konala, V.B.R., Mamidi, M.K., Bhonde, R., et al. (2016) The Current Landscape of the Mesenchymal Stromal Cell Secretome: A New Paradigm for Cell-Free Regeneration. Cytotherapy, 18, 13-24. https://doi.org/10.1016/j.jcyt.2015.10.008

[28] Fernandez, T.L., Van Lonkhuyzen, D.R., Dawson, R.A., et al. (2014) In Vitro Investigations on the Effect of Dermal Fibroblasts on Keratinocyte Responses to Ultraviolet B Radiation. Photochemistry and Photobiology, 90, 1332-1339. https://doi.org/10.1111/php.12317

[29] Le Pillouer-Prost, A. (2003) Fibroblasts: What's New in Cellular Biology? Journal of Cosmetic and Laser Therapy, 5, 232-238. https://doi.org/10.1080/14764170310021869

[30] Amaro-Ortiz, A., Yan, B. and D’Orazio, J. (2014) Ultraviolet Radiation, Aging and the Skin: Prevention of Damage by Topical cAMP Manipulation. Molecules, 19, 6202-6219. https://doi.org/10.3390/molecules 19056202

[31] Park, B.S., Jang, K.A., Sung, J.H., et al. (2008) Adipose-Derived Stem Cells and Their Secretory Factors as a Promising Therapy for Skin Aging. Dermatologic Surgery, 34, 1323-1326.

[32] OdileDamour, M. (2014) Adipose-Derived Stem Cells Promote Skin Homeostasis and Prevent Its Senescence in an in Vitro Skin Model. Journal of Stem Cell Research \& Therapy, 4, 194 
Submit or recommend next manuscript to SCIRP and we will provide best service for you:

Accepting pre-submission inquiries through Email, Facebook, LinkedIn, Twitter, etc. A wide selection of journals (inclusive of 9 subjects, more than 200 journals)

Providing 24-hour high-quality service

User-friendly online submission system

Fair and swift peer-review system

Efficient typesetting and proofreading procedure

Display of the result of downloads and visits, as well as the number of cited articles Maximum dissemination of your research work

Submit your manuscript at: http://papersubmission.scirp.org/

Or contact jcdsa@scirp.org 